Article

\title{
Multi Equipment Condition Based Maintenance Optimization Using Multi-Objective Evolutionary Algorithms
}

\author{
Aitor Goti ${ }^{1, *,+}\left(\mathbb{D}\right.$, Aitor Oyarbide-Zubillaga ${ }^{2,+}$, Ana Sanchez ${ }^{3,+}$, Tugce Akyazi ${ }^{2,+}$ and \\ Elisabete Alberdi ${ }^{4,+}$ \\ 1 Deusto Digital Industry Chair, University of Deusto, 48007 Bilbao, Bizkaia, Spain \\ 2 Department of Mechanics, Design and Organization, University of Deusto, 48007 Bilbao, Bizkaia, Spain; \\ aitor.oyarbide@deusto.es (A.O.-Z.); tugceakyazi@deusto.es (T.A.) \\ 3 Statistics and Operational Research and Quality Department, Polytechnic University of Valencia, \\ 46022 Valencia, Spain; aisanche@eio.upv.es \\ 4 Department of Applied Mathematics, University of the Basque Country UPV/EHU, \\ 48013 Bilbao, Bizkaia, Spain; elisabete.alberdi@ehu.eus \\ * Correspondence: aitor.goti@deusto.es; Tel.: +34-944-139-000 \\ + These authors contributed equally to this work.
}

Received: 9 October 2019; Accepted: 7 November 2019; Published: 13 November 2019

\begin{abstract}
Thanks to the digitalization of industry, maintenance is a trending topic. The amount of data available for analyses and optimizations in this field has increased considerably. In addition, there are more and more complex systems to maintain, and to keep all these devices in proper conditions, which requires maintenance management to gain efficiency and effectiveness. Within maintenance, Condition-Based Maintenance (CBM) programs can provide significant advantages, but often these programs are complex to manage and understand. The problem becomes more complex when equipment is analyzed in the context of a plant, where equipment can be more or less saturated, critical regarding quality, etc. Thus, this paper focuses on CBM optimization of a full industrial chain, with the objective of determining its optimal values of preventive intervention limits for equipment under economic criteria. It develops a mathematical plus discrete-event-simulation based model that takes the evolution in quality and production speed into consideration as well as condition based, corrective and preventive maintenance. The optimization process is performed using a Multi-Objective Evolutionary Algorithm. Both the model and the optimization approach are applied to an industrial case, where the data gathered by the IoT (Internet of Things) devices at edge level can detect when some premises of the CBM model are no longer valid and request a new simulation. The simulation performed in a centralized way can thus obtain new optimal values who fit better to the actual system than the existing ones. Finally, these new optimal values can be transferred to the model whenever it is necessary. The approach developed has raised the interest of a partner of the Deusto Digital Industry Chair.
\end{abstract}

Keywords: Condition-Based Maintenance; Genetic Algorithms

\section{Introduction}

Maintenance has always been a relevant topic for operations [1,2]. Nowadays, the importance of maintenance is increasing thanks to "Industry 4.0" or the "fourth industrial revolution" [3], which promotes automation through computer systems in manufacturing and aims to achieve intelligent or smart factory. Several studies propose the simplest possible approaches that can be understood by users and modified by experts [4,5]. Nevertheless, industrial plan management, and particularly 
maintenance optimization, needs to consider several parameters that are not measurable by the same standard and respond to contradictory objectives. It is possible to over or under extend the operational lifetime of the equipment, by increasing or reducing preventive maintenance (PM) expenditures and thus preventing catastrophic failures, but without taking into account the overall plant profitability. Therefore, maintenance has a critical importance during production. Since maintenance requirements depend on a high amount of parameters, it is very delicate to regulate the optimal maintenance strategy that maximizes the profitability of the equipment. In addition, equipment focused long-term profitability is usually de-aligned with the short-term needs of directors, making decisions even more difficult.

In recent years, many studies from different points of view have been carried out to find an optimal Condition Based Maintenance (CBM) policy. They were mostly focused on the optimization of single deteriorating equipment and did not consider either the configuration of the productive system or the influence of the whole system on each studied machine. Thus, although single equipment optimization methods might be useful in the case of productivity bottlenecks or continuous processes, they are less convenient in the case of machines working in multi-equipment manufacturing systems.

Maintenance requirements of a single machine belonging to a multi-equipment system are directly determined by the amount of the stock of semi-elaborated products related to the machine as well as on the machine being a bottleneck or not. For example, the availability of a bottleneck machine will be critically important for the profitability of the company, whereas its failure will not have such a significant impact on the whole system (if stock levels and repair times are under control). Nevertheless, the recent literature (as summarized in specific reviews [6-8]) shows that the joint optimization of CBM activities of multi-equipment systems is still an area lacking sufficient research.

In this work, we present an innovative solution for the joint optimization of CBM strategies applied on several machines belonging to a productive system. The study aimed to find the optimal component deterioration levels when PM is carried out for multi-equipment systems. Therefore, a new model was developed considering maintenance, productive speed loss and non-quality costs as well as productive profit. The model was implemented using the suitability of Discrete Event Simulation (DES) and optimized using the aptitude of a Multiobjective Evolutionary Algorithm (MOEA) to reach optimal solutions.

The strength of this model is that, with the help edge computing IoT devices, it can detect when some basic parameters and assumptions of the model (e.g. Weibull data, if a machine is a bottleneck always running or not, etc.) become de-aligned with reality, and if they do so they can request a calibration (a new simulation for the optimization). This idea is presented as a conclusion and future guideline of the research in a new working scheme.

This paper is arranged as follows. The optimization problem is described in Section 2 while the developed DES plus the analytical model is summarized in Section 3. The optimization procedure is explained with detail in Section 4, whereas the results obtained and conclusions are presented in Section 5 .

\section{Optimization Problem}

In this work, a new method was developed to optimize CBM activities of a simplified multi-equipment hub cap production system. The work is based on the multi-component but single equipment model presented in [9]; as added value, this research extends it using DES so that in this case it is possible to perform a multi-equipment optimization. In previous studies [10,11], a PM optimization [10] and a CBM optimization based on a different deterioration model [11] were presented. In the case of Valčuha etal. [11], they applied it for the same system but using a different ageing model, whereas Goti, Oyarbide-Zubillaga and Sanchez [10] optimized preventive maintenance instead of CBM. This system is set up in a company of the Mondragon Corporation. As shown in Figure 1, the production system is made up of three identical plastic injection machines and a painting station and it generates plastic made hub caps for car-maker companies. 


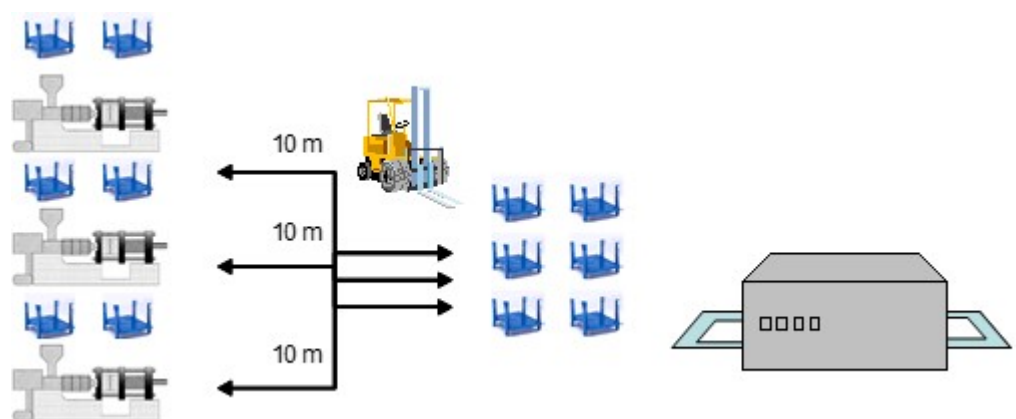

Figure 1. A representation of the simplified plastic injection system.

In the studied production system, the manufacturing process starts by injecting and compressing the plastic contemporaneously in the injection machine. Then, once it is cooled, the mold is opened, and the injected product is extracted. Later, the final product is located along the side of the injection machine buffer which consists of two pallets of 100 hubcaps each.

When a pallet is filled with hub caps, it is transported from the injection machine buffer to the painting station buffer. Then, the products are loaded onto a conveyor that leads to the painting station. At the painting station, the products are painted. Finally, they are sent to a warehouse.

Each machine belonging to the model is formed of three subsystems, which are designed as components and constructed in serial configuration.

One maintenance activity is implemented on each subsystem to control its aging: M1, M2 and M3 are, respectively, executed over sub-systems S1, S2 and S3 of the injection machines, whereas M4, M5 and M6 are sequentially applied on sub-systems S4, S5 and S6 of the painting station. Table 1 represents the influence of each subsystem on the performance of each machine: regarding the injection machine, S1's deterioration has an effect only on unavailability, S2's deterioration has an influence on unavailability and productive speed loss, and S3's deterioration affects quality and unavailability. In the case of the painting station, S4's deterioration affects only unavailability, S5's deterioration has an effect on productive speed loss and unavailability, and S6's deterioration has an influence on quality and unavailability.

Table 1. Components, PM activities and their influences.

\begin{tabular}{cccc}
\hline Equipment & Subsystem & PM Activities Performed & Influences on \\
\hline Injection machines & M1 & S1 & Unavailability \\
& M2 & S2 & Unavailability and Productive speed loss \\
& M3 & S3 & Unavailability and Quality \\
\hline Painting station & M4 & S4 & Unavailability \\
& M5 & S5 & Unavailability and Productive speed loss \\
& M6 & S6 & Unavailability and Quality \\
\hline
\end{tabular}

Regarding the equipment failure process, a two-parameter $\left(\lambda_{1}\right.$ and $\left.\gamma_{1}\right)$ Weibull failure rate is used for the modeling. The Weibull distribution was chosen for developing this model because it is the most common distribution to model reliability data. In addition, during the modeling, a process deterioration which shifts the system from an under-control state to an out-of-control state is taken into account. This process of deterioration acts in accordance with a Weibull distribution of parameters $\lambda_{2}$ and $\gamma_{2}$. The Weibull reliability data for the studied problem are demonstrated in Table 2. 
Table 2. Weibull data of the studied subsystems.

\begin{tabular}{|c|c|c|c|c|}
\hline Group & $\begin{array}{c}\lambda_{1} \\
\left(10^{-2} h^{-1}\right)\end{array}$ & $\gamma_{1}$ & $\begin{array}{c}\lambda_{2} \\
\left(10^{-2} h^{-1}\right)\end{array}$ & $\gamma_{2}$ \\
\hline S1 & 5 & 2 & & \\
\hline S2 & 2 & 2.9 & & \\
\hline S3 & 4 & 2 & 4 & 2 \\
\hline S4 & 6.6 & 2 & & \\
\hline S5 & 7.7 & 3 & & \\
\hline S6 & 10 & 3 & 110 & 3 \\
\hline
\end{tabular}

\section{Discrete Event Simulation Model}

DES deals with the modeling of a system, which evolves with time using a model where variable states alter drastically at different points of time. These alterations occurring in the system are named as events. Since systems do not change in the middle of events, DES model does not find it necessary to investigate the circumstances of a system during the time interval between two events.

There are two main benefits of DES. Firstly, standard DES-based tools enable to have modeling capabilities or to modify complex system models in a very simple manner. Secondly, DES is closely linked with stochastic systems which are ideally used for simulating real-world phenomena. In several situations, using stochastic systems, it is possible to predict the actions of the entities beforehand. Additionally, it is possible to carry out the stochastic nature of real models in DES models, using pseudo-random numbers.

In this work, we used the DES model to simulate the injection machines, the painting station, the lift, the product buffers and the pallets. The application of the model on each of the components is described in detail in the following subsections.

\subsection{Equipment Modeling}

In the DES model, the behavior pattern of the machines is described by an analytical model. A previous research developed by Goti and Sanchez [12] considered this analytical model as a single equipment model. The present work takes into account the modeling of maintenance, quality and production speed loss costs simultaneously, with the benefit related to the production of non-defective products. The decision variables $(\mathbf{x})$ are the critical age levels when PM activities are carried out; thus, CBM deterioration thresholds, along with instants when a PM activity is not enough and a substitution of the component is needed (the As Good As New (GAN) model). They are optimized considering cost and profit criteria, and they affect directly all the terms mentioned above (modeling of maintenance, quality, production speed loss costs, etc.).

The aforementioned equipment model is built under the following assumptions: (1) An imperfect maintenance model determines the effect of the maintenance activities. In such cases, a Proportional Age Reduction (PAR) [13] is considered. (2) The deterioration and failure processes are independent from each other. (3) When the process is out-of-control, the system only delivers non-conforming components, with a rate constant $(\alpha)$. (4) Preventive maintenance and process inspection are carried out concurrently. (5) Inspections are free of errors. (6) When the preventive maintenance is performed, the process is brought back to under control state. (7) Productive speed drops from its initial speed $\left(V_{0}\right)$ to another speed value $\left(V^{*}(\mathbf{x})\right)$, which is directly related to the CBM decision variables $\left.(\mathbf{x})\right)$. (8) All deterioration processes related to the three studied components are independent. (9) Since the process delivers only one type of product, setup times of reference changes are not calculated. Unlike the Condition Monitoring (CMT) of the component, done continuously, PM is only performed when a component has a specific critical age or deterioration level $\left(w_{c}\right)$. Moreover, PAR model assumes that the maintenance reduces the age of the component proportionally by a $\varepsilon$ factor, between the moment after executing the previous PM activity $\left(w_{m-1}^{+}\right)$and the moment before the last one $\left(w_{m}^{-}\right)$. The assumptions 
to obtain the expressions for the PAR model are the same as in [12]. The results of these assumptions are stated in Figure 2, where the age of a component $w$ is compared with its chronological time.

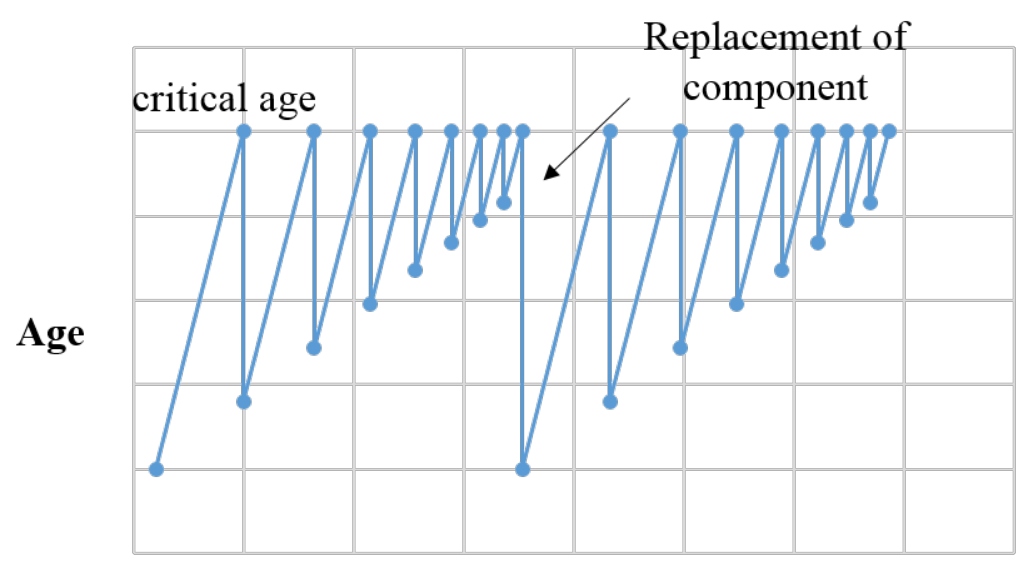

\section{Chronological time}

Figure 2. Age vs. chronological time in a PAR model under a CM maintenance strategy [12].

Figure 2 shows that the component is continuously monitored, i.e. no preventive action is carried out until it reaches a critical age $w_{c}$. It can be observed that corrective maintenance is performed with minimal repair (the As Bad As Old (BAO) model is adopted), and PM is modeled considering a PAR model with effectiveness $\varepsilon$. Moreover, the time between two PM activities, M, gets shorter and shorter, following this equation:

$$
M_{m}=w_{c} \cdot \varepsilon^{m-1} .
$$

This makes is necessary to fix a limit value on time between two PM activities, $M_{\text {min }}$, from which the component will be upgraded with an intervention of $\varepsilon=1$ (GAN). The aim of the problem is to optimize the limit values $M_{\min }$ and the values $W_{c}$. Hence, a component will be installed for a $L_{c}$ time period and then it will be upgraded (or substituted) into a GAN situation:

$$
L_{c}=\sum_{k=1}^{e} M_{k}
$$

where $e$ represents the amount of PMs executed before a component is replaced. It has to be taken into account also that during a $L$ simulation period $n_{c}$ components will be consumed:

$$
n_{c}=\frac{L}{L_{c}} .
$$

With these assumptions, the next the features of the economic model are presented in the following subsections.

\subsubsection{Maintenance costs}

Different types of costs have to be considered: costs due to condition monitoring (CMT); preventive maintenance (PM); corrective maintenance (CM); consequence of idling, minor stops and failure or breakdowns; and costs of upgrading the components or substituting them $\left(C_{u}\right)$. Each of them is defined next.

The cost associated to condition monitoring $\left(C_{c m t}\right)$ is given by:

$$
C_{c m t}=L \cdot C_{h c t} .
$$


$L$ represents the analysis period and $C_{h c t}$ is the hourly cost of monitoring. Preventive maintenance costs are calculated following:

$$
C_{p m}=n_{c} \cdot e \cdot d_{p m} \cdot c_{h p m}
$$

where $d_{p m}$ and $c_{h p m}$ represent, respectively, the mean time and the average hourly cost of performing $\mathrm{PM}$, and $\mathbf{x}$ is the vector of decision variables. It is worth noting that this cost will be incurred in every PM action in this model. In addition, some preventive actions will require adding the cost of upgrading the component.

The cost contribution due to corrective maintenance is given by:

$$
C_{c m}(\mathbf{x})=u_{c m}^{*}(\mathbf{x}) \cdot c_{h c m} \cdot L,
$$

where $c_{h c m}$ is the average hourly cost of performing corrective maintenance, and $u_{c m}^{*}(\mathbf{x})$ is the averaged unavailability of the analysis period $L$. The calculation of $u_{c m}^{*}(\mathbf{x})$ requires knowing $u_{r}^{*}(\mathbf{x})$, the time-dependent unreliability for discontinuous equipment, which takes the expression of $u_{r m}^{*}(\mathbf{x})$ each period between PM activities:

$$
u_{r m}^{*}(\mathbf{x})=\rho+(1-\rho) \cdot\left(1-\exp \left(-h_{m}^{*} \cdot M_{m}\right)\right) .
$$

Thus, it is possible to obtain $u_{c m}^{*}(\mathbf{x})$ :

$$
u_{c m}^{*}(\mathbf{x})=\frac{\sum_{m=1}^{e} u_{r m}^{*}(\mathbf{x}) \cdot d_{c m}}{L_{c}} .
$$

Finally, we define the costs of upgrading the component $\left(C_{u}\right)$ when the amount of maintenance activities prior to change is exceeded. This term is calculated by multiplying the number of components that have been changed $\left(n_{c}\right)$ with the cost of upgrading or substituting one component $\left(C_{c}\right)$ :

$$
C_{u}=C_{c} \cdot n_{c} .
$$

\subsubsection{Costs Related to Reduced Speed Due to Aging}

The equipment model assumes that the production rate falls proportionally in relation with the age of a component following a factor $\tau$ :

$$
V_{m}(w)=V_{0}-\tau^{2} \cdot w_{m}(t, \varepsilon) .
$$

In the same way as $\mathrm{CM}$, an averaged production speed for the lifetime of a component, $V^{*}$, is calculated:

$$
V^{*}=\frac{\sum_{m=1}^{e} V_{m}^{*} \cdot M_{m}}{M_{m}} .
$$

Adopting this value of the speed production average $V^{*}$, the "production time lost" related to a reduced speed $\left(t_{s l}\right)$ can be calculated. If only a fraction of the production system is considered, the production time lost is available as follows:

$$
t_{s l}(\mathbf{x})=L \cdot\left(1-A_{s}(\mathbf{x}) \cdot \frac{V^{*}}{V_{0}}\right),
$$

where $A_{s}(\mathbf{x})$ is the availability system resulting from the combination of the unavailabilities of the components. To finish with the different type of costs, the ones related to the production speed loss of the equipment $\left(C_{s l}\right)$ in the period $L$ can be evaluated proportionally to the production time lost:

$$
C_{s l}(\mathbf{x})=C_{h s l} \cdot t_{s l}(\mathbf{x}),
$$


where $C_{h s l}$ is the average hourly cost due to non-produced items. By substituting Equation (12) into Equation (13), we have:

$$
C_{s l}(\mathbf{x})=C_{h s l} \cdot L \cdot\left(1-A_{s}(\mathbf{x}) \cdot \frac{V^{*}}{V_{0}}\right)
$$

\subsubsection{Quality Costs}

Quality costs are obtained considering the effects on PM and upgrading activities on the component age based on the PAR model. The developed model assumes that: (1) The equipment only produces non-conforming items with constant rate, $\alpha$, while the process is out-of-control. (2) The time to the system swaps out-of-control follows a Weibull distribution, which depends on the age of the equipment. (3) The preventive maintenance and the process inspection are executed simultaneously. (4) Inspections do not have errors. (5) The process is restored to the in-control state when the preventive maintenance activity is carried out. To model the quality costs, it is crucial to determine the fraction of time during which the process is under control (UC) state named $\kappa_{m}(w)$.

$$
\kappa_{m}(w)=\int_{w_{m-1}^{+}}^{w_{m}^{-}} w_{m} \cdot f\left(w_{m}\right) \cdot d w_{m},
$$

where $f\left(w_{m}\right)$ is the density function, which can be obtained using the conditional hazard function of the component, as detailed in the following equation:

$$
f\left(w_{m}\right)=\lambda \cdot \gamma\left(\lambda \cdot w_{m}\right)^{\gamma-1} \exp \left(-\left(\lambda \cdot w_{m}\right)^{\gamma}\right),
$$

being $w_{m-1}^{+}=w_{c}(1-\varepsilon) \sum_{k=1}^{m-1} \varepsilon^{k-1}$ and $w_{m}^{-}=w_{c}$.

Thus, it is possible to obtain the time with the process under control between two maintenance activities $\left(M_{m}-\kappa_{m}\right)$, as well as the quality costs, $C_{q}$ as:

$$
C_{q}=n_{c} \cdot C_{\alpha} \cdot \sum_{m=1}^{e} V_{m}^{*} \cdot\left(M_{m}-\kappa_{m}\right) \cdot A(\mathbf{x}) \cdot \alpha,
$$

being $C_{\alpha}$ the cost of the non-conforming unit.

\subsubsection{Profit}

To evaluate the consequences of a given preventive maintenance schedule in economic terms, it is crucial to consider the costs as well as the benefits obtained by its implementation. Thus, a net profit function, $P$, denoting the benefits obtained to the sale of products, is introduced as:

$$
P=n \cdot \Psi \text {. }
$$

\subsection{Embedment of the Analytical Equipment Models into DES}

In this work, to achieve stochastic events making equipment function equally (as it is specified in the analytical model), analytical formulation related to each machine in the productive system is applied on the equipment. To carry out the integration process, first the analytical evaluation of the components of the decision vector corresponding to the studied machines is performed. Through this process, the working parameters $A(\mathbf{x}), V^{*}(\mathbf{x})$ and $\kappa^{*}(\mathbf{x})$ of the corresponding $w_{c}$ and $M_{\min }$ values are generated. Finally, the obtained working parameters are presented as inputs in the DES modeled machines in order to carry out the simulation for the optimization of the results.

The whole integration process leads to the generation of $\mathrm{PM}$, unplanned $\mathrm{CM}$, speed reduction and defective product events and actions during the simulation. As a result, the same operative values of the analytical model are produced by the machines. As noteworthy input, the DES model models the features of a single machine as well as the interaction among the machines, since it is capable 
of combining different machines in a production system. The costs of the mentioned events must also be considered since the generation of each event is related to a particular inefficiency. Costs are determined taking into account CM, PM, speed loss, quality and profit terms using counters. When the simulation is started, these counters are set to zero and each time an event related to them is created by the simulation, the counter values are increased accordingly.

\subsection{Buffer and Transportation Modeling}

System buffers have a certain capacity. The model accepts that, if a buffer is filled with products, it will not accept more products until the pallets are unloaded (the transportation events will not be carried out). In other words, if a machine does not have any place to drop the products, it will stop production.

Each of the injection machines delivers to a buffer of two pallets, each containing 100 products, then a buffer of ten pallets feeds the painting station.

Regarding transportation modeling, semi-elaborated product movements were modeled exclusively. During the modeling process, movements between the following were considered: (i) a machine and a buffer location; (ii) two machines; (iii) a buffer location and a machine; and (iv) two buffer locations. To mention transportation types, for Movements (i)-(iii), products are transported by the piece, while, for Movement (iv), products are moved using pallets. During the modeling process of these movements, a delay is introduced in the system. Therefore, at time $t$, the element is at the initial point, whereas, at time $t+d e l a y$, it is at the destination point. To simplify the whole process, Movements (i)-(iii) are not modeled (delay $=0$ ). Empty pallets are inserted into the system (and removed out of it) to transport products automatically and immediately. The lift truck transport is modeled with a delay having a uniform distribution range between 14.4 and $28.8 \mathrm{~s}$.

\subsection{Simulation Values of the Productive System}

In the next four tables, data collected for the simulation model are demonstrated in detail. Tables 3 and 4 present parameters related to PM and CM, while Tables 5 and 6 show data about inputs related to $\mathrm{CM}$, speed, quality, unavailability, and cost for the injection machines and the painting station. In these tables $u$ represents a manufactured product unit. Thus, the unavailability of the machine will be affected by a mixture of different ages of all the subsystems.

Table 3. PM data related to the productive system.

\begin{tabular}{ccc}
\hline Activity Maintenance & $\varepsilon$ & $\boldsymbol{d}_{\boldsymbol{p m}}(\boldsymbol{h})$ \\
\hline M1 & 0.9 & 0.5 \\
M2 & 0.9 & 1 \\
M3 & 0.9 & 1 \\
M4 & 0.9 & 2 \\
M5 & 0.9 & 1 \\
M6 & 0.9 & 3 \\
\hline
\end{tabular}

Table 4. CM data related to the productive system.

\begin{tabular}{cc}
\hline $\begin{array}{c}\text { Corrective Breakdown } \\
\text { of Subsystem }\end{array}$ & $\boldsymbol{d}_{\boldsymbol{p} m}(\boldsymbol{h})$ \\
\hline S1 & 0.5 \\
S2 & 1 \\
S3 & 2 \\
S4 & 0.5 \\
S5 & 1 \\
S6 & 2 \\
\hline
\end{tabular}


Table 5. Productive and cost parameters for the injection machines.

\begin{tabular}{ccccccccc}
\hline $\begin{array}{c}C_{\alpha} \\
€ / u\end{array}$ & $\begin{array}{c}\tau \\
\boldsymbol{u} / h^{2}\end{array}$ & $\begin{array}{c}C_{\text {hsl }} \\
€ / h\end{array}$ & $\begin{array}{c}\rho \\
\mathbf{1 0}\end{array}$ & $\alpha$ & $\begin{array}{c}h_{0} \\
\text { fail } / \boldsymbol{h}\end{array}$ & $\begin{array}{c}V_{0} \\
\boldsymbol{u} / \boldsymbol{h}\end{array}$ & $\begin{array}{c}c_{\text {hcm }} \\
€ / h\end{array}$ & $\begin{array}{c}c_{\text {hpm }} \\
€ / h\end{array}$ \\
\hline 6 & 0.0017 & 25 & 1 & 0.03 & 0 & 180 & 45 & 30 \\
\hline
\end{tabular}

Table 6. Productive and cost parameters for the painting station.

\begin{tabular}{cccccccccc}
\hline $\begin{array}{c}C_{\alpha} \\
€ / u\end{array}$ & $\begin{array}{c}\tau \\
u / h^{2}\end{array}$ & $\begin{array}{c}C_{h s l} \\
€ / h\end{array}$ & $\begin{array}{c}\rho \\
\mathbf{1 0}^{-3}\end{array}$ & $\alpha$ & $\begin{array}{c}h_{0} \\
\text { fail/h }\end{array}$ & $\begin{array}{c}V_{0} \\
u / h\end{array}$ & $\begin{array}{c}c_{h c m} \\
€ / h\end{array}$ & $\begin{array}{c}c_{h p m} \\
€ / h\end{array}$ & $\begin{array}{c}c_{h c b m} \\
€ / h\end{array}$ \\
\hline 6 & 0.02 & 150 & 1 & 0.04 & 0 & 900 & 175 & 160 & 2 \\
\hline
\end{tabular}

In addition to all the data provided, the net profit value of a non-defective product ( $\Psi)$ is evaluated as $0.2 €$ /unit and the simulation time $L$ is considered as 62,400 working hours, which corresponds to 10 years of production (in the case of working five days a week and $24 \mathrm{~h}$ a day). Eventually, products are manufactured in batches of 100 units.

\section{Optimization Procedure}

In this work, the productive costs and profit are considered as optimization criteria during the optimization process of deterioration levels while PM activities were set in motion. Both profit and cost models depend on the decision vector, $\mathbf{x}$. Thus, the vector of bi-objective function, $f(\mathbf{x})$, is described as:

$$
\mathbf{f}(\mathbf{x})=(C(\mathbf{x}), P(\mathbf{x})),
$$

where the aim is to make the function $C(\mathbf{x})$ minimum, and the profit function $P(\mathbf{x})$ maximum. $C(\mathbf{x})$ is defined as the cost system function, which is calculated as sum of the maintenance, quality costs, and production speed lost for each machine of the system. $P(\mathbf{x})$ is the profit function extracted from the sale of non-defective products. In this case, there are no limitations present in the vector of constraints. However, there are constraints present in the values that the decisions variables can take, which must be typified, representing each one a day, two days, etc.

This maintenance optimization problem was approached using the NSGA-II algorithm [14], a still very efficient MOEA for bi-objective optimization problems that tends to achieve solutions which are located on a Pareto optimal front, where it cannot be concluded that a solution obtained is better than another without considering additional information.

\section{Results and Conclusions}

Figure 3 presents a cost-benefit plot of results found by the NSGA-II, with a selection rate of 0.25 , crossover rate of 0.5 and mutation rate of 0.75 (based on the values used in [15]), evolving a population of 50 individuals for 400 generations.

Once the solutions are obtained, the possible additional restrictions related to them can be taken into consideration concurrently. This situation gives the decision maker the opportunity of selecting the best maintenance strategy externally, combining short-term business profitability and long-term asset management strategy terms.

Another enriching feature of the developed approach is that, after defining "a posteriori" decision criteria, it is possible to assign optimal CBM levels to items, monitor them through edge IoT devices. In addition, it can detect through edge computing devices that the expected behavior of the system is not the one simulated, and then simulations can be run back again to re-optimize the system. The holistic view of the initiative is shown in Figure 4. 


\section{Cost and benefit optimization results}

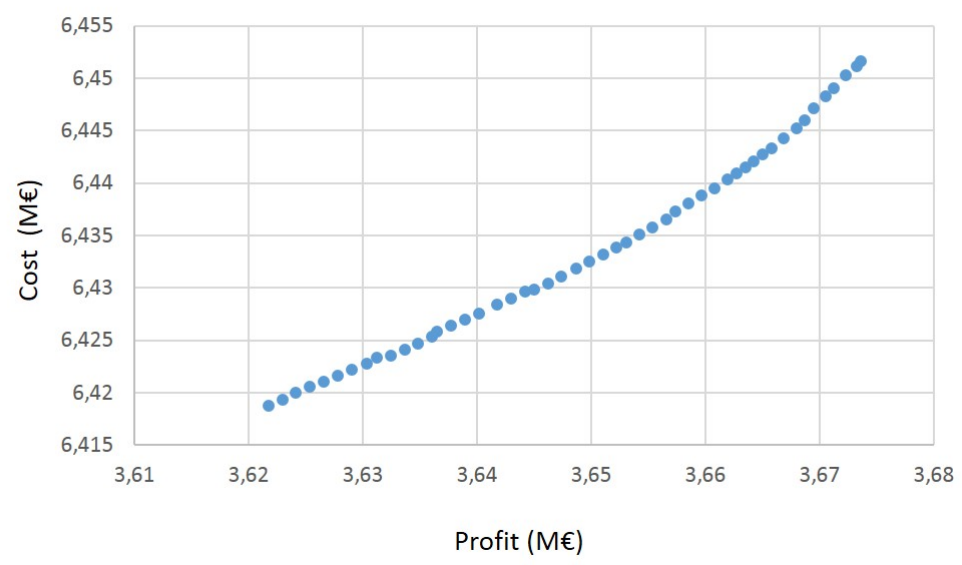

Figure 3. Results of the optimization.

It is worth remarking that the approach has raised the interest of a company within the Deusto Digital Industry Chair. Therefore, the efforts of the chair will be oriented to consolidate this initiative.

\section{EDGE LEVEL}

Detect at edge level and communicate via loT:

- changes in the best fitting ageing models and deterioration parameters changes in the productive configuration (lay-out, bottleneck...) Suggest new optimization

FOG/CLOUD LEVEL

Request review of economic parameters to managers

Simulate to find again:

- best fitting ageing models and deterioration parameters

- Optimal CBM values

\section{EDGE LEVEL}

Implement at edge level: - new optimal CBM values

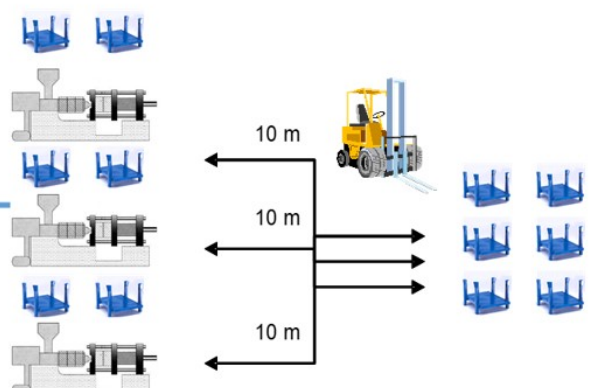

प्र००

Figure 4. CBM optimization scheme of the project approach.

Author Contributions: A.G., writing, conceptualization and investigation; A.O.-Z. and A.S., conceptualization and investigation; and T.A. and E.A., writing and investigation.

Funding: This research was funded by the HAZITEK call of the Basque Government, project acronym HORDAGO.

Acknowledgments: We would like to thank the partners of the Deusto Digital Industry Chair (Etxe-Tar, General Electric, Idom, Accenture, Fundación Telefónica, and Fundación BBK) for the interest and support shown during this research.

Conflicts of Interest: The authors declare no conflict of interest. 


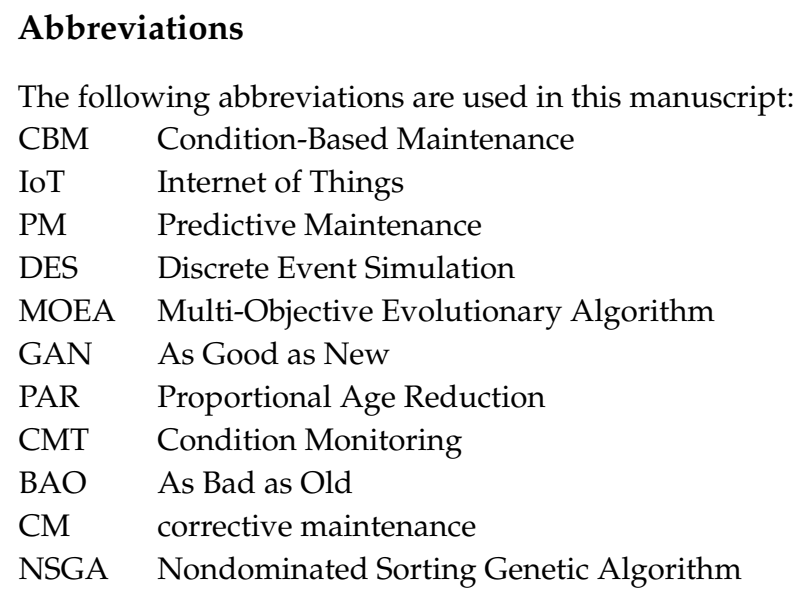

\section{References}

1. Goti-Elordi, A.; Egaña-Errasti, M.M.; de Albéniz, A.I.-P. Encuesta sobre el estado del mantenimiento industrial en España. Dyna 2009, 84, 225-230.

2. Unzueta-Aranguren, G.; Goti-Elordi, A.; Garitano-Aranda, J.; Sánchez-Ganchegui, I. Aplicación de un sistema de gestión del mantenimiento basado en un RCM adaptado. Dyna 2014, 89, 347-354.

3. Suarez-García, A.; Rey, G.; Arce-Fariña, E.; Alvarez-Feijoo, M. Spanish navy and Reliability Centered Maintenance. Dyna 2018, 93, 580-587.

4. de Jonge, B.; Teunter, R.; Tinga, T. The influence of practical factors on the benefits of condition-based maintenance over time-based maintenance. Reliab. Eng. Syst. Saf. 2017, 158, 21-30. [CrossRef]

5. Parrondo, J.L.; Velarde, S.; Santolaria, C. Development of a predictive maintenance system for a centrifugal pump. J. Qual. Maint. Eng. 1998, 4, 198-211. [CrossRef]

6. Alaswad, S.; Xiang, Y. A review on condition-based maintenance optimization models for stochastically deteriorating system. Reliab. Eng. Syst. Safe. 2017, 157, 54-63. [CrossRef]

7. Bousdekis, A.; Magoutas, B.; Apostolou, D.; Mentzas, G. Review, analysis and synthesis of prognostic-based decision support methods for condition based maintenance. J. Intell. Manuf. 2015, 1, 1-14. [CrossRef]

8. Kang, J.; Sobral, J.; Guedes Soares, C. Review of Condition-Based Maintenance Strategies for Offshore Wind Energy. J. Mar. Sci. Appl. 2019, 18, 1-16. [CrossRef]

9. Goti, A.; Oyarbide-Zubillaga, A.; Alberdi, E.; Sanchez, A.; Garcia-Bringas, P. Optimal Maintenance Thresholds to Perform Preventive Actions by Using Multi-Objective Evolutionary Algorithms. Appl. Sci. 2019, 9, 3068. [CrossRef]

10. Goti, A.; Oyarbide-Zubillaga, A.; Sanchez, A. Optimizing preventive maintenance by combining discrete event simulation and genetic algorithms. Hydrocarb. Process. 2007, 86, 115-122.

11. Valčuha, Š.; Goti, A.; Úradníček, J.; Navarro, I. Multi-equipment condition based maintenance optimization by multi- objective genetic algorithm Analysis and modelling. J. Achiev. Mater. Manuf. Eng. 2011, 2, 188-193.

12. Goti, A.; Sanchez, A.I. Condition-based Maintenance: Determination of optimal deterioration levels to perform preventive activities by using a multi- objective evolutionary algorithm. In Proceedings of the European Conference of the PHM Society 2016 Proceedings, Bilbao, Spain, 5-8 July 2016.

13. Malik, M.A.K. Reliable preventive maintenance scheduling. AIIE Trans. 1979, 11, 221-228. [CrossRef]

14. Deb, K.; Pratap, A.; Agarwal, S.; Meyarivan, T. A fast and elitist multiobjective genetic algorithm: NSGA-II. IEEE Trans. Evolut. Comput. 2002, 6, 182-197. [CrossRef]

15. Oyarbide-Zubillaga, A.; Sanchez, A.; Goti, A. Preventive maintenance optimisation of multi-equipment manufacturing systems by combining discrete event simulation and multi-objective evolutionary algorithms. Prod. Plan. Control. 2008, 19, 342-355. [CrossRef]

(C) 2019 by the authors. Licensee MDPI, Basel, Switzerland. This article is an open access article distributed under the terms and conditions of the Creative Commons Attribution (CC BY) license (http:/ / creativecommons.org/licenses/by/4.0/). 